\title{
Modelling crowd-structure interaction
}

\author{
Philippe Pécol ${ }^{1, a}$, Stefano Dal Pont ${ }^{2}$, Silvano Erlicher $^{3}$ And Pierre Argoul ${ }^{1}$ \\ 1 Université Paris-Est, Laboratoire Navier, École des Ponts-ParisTech, LCPC, CNRS, 6-8 Av. Blaise Pascal, Cité Descartes, \\ Champs sur Marne, 77455 Marne la Vallée Cedex 2, France \\ 2 Université Paris-Est, Laboratoire Central des Ponts et Chaussées, LCPC-BCC, 58 boulevard Lefebvre, 75732 Paris, France \\ 3 IOSIS Industries, 35 rue du Val de Marne, 75013 Paris, France
}

Received 25 February 2010, Accepted 23 April 2010

\begin{abstract}
An emerging research topic in civil engineering is the dynamic interaction between crowds and structures. Structures such as footbridges, which oscillate due to the crossing of a group of pedestrians, or stands within stadia or concert halls, which vibrate due to the rythmic movement of the audience are of particular interest. The objective of this study is twofold: modelling the movement of pedestrians with consideration of pedestrian-pedestrian, and pedestrian-obstacle interactions, and the incorporation of a pedestrian-structure coupling in the previous model. Frémond's model, which allows us to simulate the movement of an assembly of particles and accounts for collisions among considered rigid particles, is presented and adapted to the crowd by giving a willingness to the circular particles, which allows each pedestrian to move according to a given target. To handle the crowd-structure interaction in the case of lateral oscillations of footbridges, the Kuramoto differential equation governing the time evolution of the lateral motion of each pedestrian is implemented in the previous model. Preliminary results obtained from numerical simulations are presented and discussed.
\end{abstract}

Key words: Granular assembly / crowd / contact / crowd-structure interaction / synchronization / footbridge

Résumé - Modèle pour l'interaction foule-structure. Un axe de recherches émergeant dans le domaine du génie civil est l'analyse et la modélisation des effets de la foule sur les ouvrages du génie civil. Cet article traite de la modélisation de l'interaction dynamique foule-structure. Les structures particulièrement concernées sont les passerelles piétonnes qui oscillent lors du passage d'un groupe de personnes ou les gradins des stades et les salles de concert qui vibrent à cause du mouvement rythmé du public. L'objectif de cet article est double : proposer un modèle de foule qui permet de gérer les mouvements des piétons en prenant en compte les interactions locales piéton-piéton et piéton-obstacle puis réaliser un couplage piéton-structure. Pour cela, nous avons retenu un modèle fondé sur les idées de Frémond, permettant habituellement de simuler le mouvement d'une assemblée de grains et capable de gérer les collisions entre particules supposées rigides. Ce modèle est ensuite adapté à la foule en représentant les piétons par des grains circulaires rigides «actifs» (permettant à chaque piéton de choisir sa direction). Enfin, pour tenir compte du couplage piéton-structure dans le cas des oscillations latérales des passerelles piétonnes, le modèle mathématique de Kuramoto qui permet de gérer l'évolution du mouvement latéral de chaque piéton, est retenu et implémenté. Les premiers résultats obtenus avec le modèle complet, appliqué à la travée nord de la passerelle du Millenium à Londres sont présentés et commentés.

Mots clés : Assemblée de grains / foule / contact / interaction foule-structure / synchronisation / passerelle

\footnotetext{
${ }^{a}$ Corresponding author: philippe.pecol@enpc.fr
} 


\section{Nomenclature}

\begin{tabular}{|c|c|}
\hline \multicolumn{2}{|l|}{ Roman letters } \\
\hline$A$ & lateral displacement's amplitude of the structure $(\mathrm{m})$ \\
\hline$C_{\text {str }}$ & modal (generalized) damping of the first lateral mode of the structure $\left(\mathrm{kg} \cdot \mathrm{s}^{-1}\right)$ \\
\hline$C$ & pedestrians' sensitivity to bridge vibrations $\left(\mathrm{m}^{-1} \cdot \mathrm{s}^{-1}\right)$ \\
\hline$D_{j k}$ & distance between two objects $j$ and $k(\mathrm{~m})$ \\
\hline f & forces applied to the system (heap of particles, crowd of pedestrians) (N) \\
\hline$F_{j}$ & total lateral force applied by pedestrian $j$ to the structure $(\mathrm{N})$ \\
\hline$F_{\mathrm{T}, j}$ & force applied by the $j$ th pedestrian to the structure $(\mathrm{N})$, tangential to the pedestrian's motion \\
\hline$F_{\mathrm{N}, j}$ & force applied by the $j$ th pedestrian to the structure $(\mathrm{N})$, normal to the pedestrian's motion \\
\hline$i$ & imaginary unit $\left(i^{2}=-1\right)$ \\
\hline$K_{\text {str }}$ & modal (generalized) stiffness of the first lateral mode of the structure $\left(\mathrm{kg} \cdot \mathrm{s}^{-2}\right)$ \\
\hline$L$ & length $(\mathrm{m})$ \\
\hline$l$ & width $(\mathrm{m})$ \\
\hline$m$ & mass of one object $(\mathrm{kg})$ \\
\hline M & mass matrix of the objects $(\mathrm{kg})$ \\
\hline$M_{\text {str }}$ & modal (generalized) mass of the first lateral mode of the structure $(\mathrm{kg})$ \\
\hline$n$ & number of considered objects (particles or pedestrians) \\
\hline$N_{j}$ & maximum amplitude of $F_{\mathrm{N}, j}$ \\
\hline$p$ & percussions of the system (N.s) \\
\hline$q$ & coordinates of one object in the $x y$-plane \\
\hline$\dot{\boldsymbol{q}}$ or $\boldsymbol{u}$ & actual velocity of one object in the $x y$-plane $\left(\mathrm{m} . \mathrm{s}^{-1}\right)$ \\
\hline$r$ & radius of a circular object $(\mathrm{m})$ \\
\hline$T_{j}$ & maximum amplitude of $F_{\mathrm{T}, j}$ \\
\hline$u_{\mathrm{d}}$ & desired velocity of one object in the $x y$-plane $\left(\mathrm{m} . \mathrm{s}^{-1}\right)$ \\
\hline$\ddot{U}$ & lateral modal acceleration of the structure $\left(\mathrm{m} \cdot \mathrm{s}^{-2}\right)$ \\
\hline$\dot{U}$ & lateral modal velocity of the structure $\left(\mathrm{m} \cdot \mathrm{s}^{-1}\right)$ \\
\hline$U$ & lateral modal displacement of the structure $(\mathrm{m})$ \\
\hline$v$ & velocity $\frac{\boldsymbol{u}^{+}+\boldsymbol{u}^{-}}{2}\left(\mathrm{~m} \cdot \mathrm{s}^{-1}\right)$ \\
\hline \multicolumn{2}{|r|}{ (2) } \\
\hline$\Delta_{j k}(\boldsymbol{u})$ & relative deformation velocity between the $j$ th and $k$ th particles \\
\hline$\Gamma$ & order parameter \\
\hline$\Phi$ & pseudopotential of dissipation (convex function) (N.m) \\
\hline$\phi$ & lateral total phase of one object $(\mathrm{rad})$ \\
\hline$\Phi^{\text {diss }}$ & pseudopotential which allows to define the dissipative percussions (N.m) \\
\hline$\Phi^{\text {reac }}$ & pseudopotential which allows to define the reactive percussions (N.m) \\
\hline$\tau$ & relaxation time of the considered reaction $(\mathrm{s})$ \\
\hline$\psi$ & first modal form of the structure \\
\hline$\psi_{\text {str }}$ & total phase of the lateral structure's displacement (rad) \\
\hline$\omega$ & lateral angular frequency $\left(\mathrm{rad} . \mathrm{s}^{-1}\right)$ \\
\hline$\theta$ & angle between the actual velocity and the desired velocity of one object (rad) \\
\hline \multicolumn{2}{|r|}{ 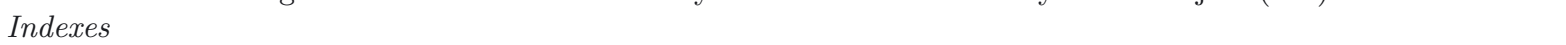 } \\
\hline 0 & at initial time $\left(t=t_{0}\right)$ \\
\hline 1 & first \\
\hline a & external acceleration \\
\hline d & desired \\
\hline$j$ & indication of the $j$ th object \\
\hline$k$ & indication of the $k$ th object \\
\hline $\bmod$ & modified \\
\hline str & structure \\
\hline step & step \\
\hline \multicolumn{2}{|l|}{ Exponents } \\
\hline+ & after a contact \\
\hline- & before a contact \\
\hline diss & dissipative \\
\hline ext & exterior \\
\hline int & interior \\
\hline reac & reactive \\
\hline$x$ & indication of the $x$-axis \\
\hline$y$ & indication of the $y$-axis \\
\hline
\end{tabular}




\section{Introduction}

\subsection{Background}

Several footbridges, recently built according to the architectural criteria of lightness and elegance, have proven to be sensitive to the excitation induced by the crossing of pedestrians. Two examples very often mentioned are the Millenium footbridge in London and the Solferino footbridge in Paris. Several measurement campaigns have allowed a better understanding of this phenomenon: the crowd walking on a bridge causes to the structure to oscillate laterally with a frequency of about $1 \mathrm{~Hz}$. When a lateral mode of vibration of the bridge, often the first, has a frequency close to this value, a resonance phenomenon is activated. It follows that the amplitude of oscillations of the bridge increases. If the number of pedestrians is small, the amplitude of oscillations stays small and pedestrians continue to walk as they would do on a rigid floor. Conversely, if this number is high enough, beyond a critical number $[1,2]$, the oscillations amplitude becomes larger, and pedestrians are forced to change their frequency of walking to prevent falling. This leads to a frequency synchronization phenomenon between pedestrians and structure. This frequency synchronization phenomenon is wellknown for lateral oscillations of a bridge [2-6], while it is not observed for the vertical direction $[2,7]$.

The behavior of a pedestrian is also influenced by the surrounding crowd: if the density of the crowd is very low, the walking is "free", i.e. each person walks as if there were nobody close to him. Conversely, if the crowd is dense, the pedestrian is forced to walk "at the same speed" as the others. This pedestrian-pedestrian synchronization can occur in both cases of rigid and moving floor. Very few studies taking simultaneously into account both types of synchronization can be found in the literature.

\subsection{State of the art}

Over the last fifty years, many studies have been performed to describe the behavior of walking pedestrians $[8,9]$. To reproduce some observed phenomena (counter flow lines, formation of arches, etc.), several models of crowd movements have been developed. These models can be classified according to different criteria: (i) the mode of representation of the crowd with macroscopic models where the crowd is represented as a whole $[6,10]$ or with microscopic models [11-17] when the behavior, the actions and the decisions of each crowd member are treated individually; (ii) the representation of the area of displacement, either continuous [11,13-17] or discretized space [12]; (iii) the representation of the contact, either by using regularizing laws [13-15] or by solving a local non linear problem [11]; (iv) the representation of pedestrian movement by means of rules $[11,12,16]$, data $[17,18]$ or forces $[13-15]$; (v) the target phenomena to be analyzed, either melees $[11,15]$ or counter flow lines $[11,13-15,17]$ or evacuation $[11,14,15]$; (vi) the type of crowd walking, either normal walking velocity [13$15,17,18]$ or emergency walking $[11,14,15]$; etc.

Concerning the pedestrian-structure coupling, some authors started modelling the dynamic loading of a single pedestrian on a vibrating structure. The human body can be seen as a very complex mechanical system, composed of several parts in mutual interactions [19]. It is therefore necessary to find a good compromise between the requirement of simplicity of the model of pedestrian and the requirement to properly reproduce a complex phenomenon such as synchronization between pedestrians and structure. Erlicher et al. [20] proposed a self-sustained single degree of freedom oscillator able to accurately predict the lateral walking force. Abrams [5] represented the action of a pedestrian on a bridge with a sinusoidal lateral force with an amplitude of $35 \mathrm{~N}$ and the evolution of its total phase is managed by a differential equation of Kuramoto. Bodgi [1] was inspired by this equation to achieve an original model of pedestrian-structure coupling.

The aim of this paper is to build a 2D crowd-structure interaction model, able to reproduce the synchronization effect due to the pedestrian-footbridge coupling. The model can also be used to study the problems of evacuation of stadia and concert halls.

This paper is divided in two parts. The first one is devoted to a brief presentation of a discrete $2 \mathrm{D}$ model for the crowd using a discrete element method to manage the movement of pedestrians in a $x y$-plane. The second part deals with the enhancement of the previous model by adding the pedestrian-structure coupling equations. The coupled model is then detailed and finally applied to the case of the Millenium bridge.

\section{2D discrete crowd model}

The approach proposed is discrete, i.e. a "microscopic" model of crowd is chosen, in which the movement of every pedestrian is represented in time and in space. The interactions of one pedestrian with the surrounding environment (other pedestrians, obstacles, moving floor) are treated locally.

We propose to model the contact among pedestrians with a granular medium model. A granular medium is by definition a set of particles that are submitted to external forces (e.g. the gravitational force), and interact each other by contacts with or without friction and with or without cohesion. Most of models which are able to deal with multiple simultaneous collisions, can be classified into two categories according to the way the contact is treated: the regular "smooth" methods [21-24] where the contact forces arise by a direct calculation, and the non regular "non-smooth" methods [25-32], in the line of the works of Moreau [33,34], where the calculation of contact forces is given by the solution of a nonlinear minimization problem.

In this paper, it has been chosen to treat the contact problem by a non-smooth model based on the theory of Frémond $[26,32]$. This model is able to consider elastic 
or inelastic collisions, to add regular forces acting on the system (for instance due to interactions without contact) and to represent an object by an arbitrary form (circular, polygonal, elliptical).

\subsection{Frémond's model}

Let us consider a system consisting of $n$ circular particles, of center ${ }^{t} q_{j}(t)=\left(q_{j}^{x}(t), q_{j}^{y}(t)\right) \in R^{2}$ and radius $r_{j}$ for the particle $j$. The velocity of the $j$ th particle is noted: $u_{j}(t)=\frac{\mathrm{d} q_{j}(t)}{\mathrm{d} t}$. The relative deformation velocity between the $j$ th and $k$ th particles is also introduced: $\boldsymbol{\Delta}_{j k}(u(t))=u_{j}(t)-u_{k}(t)$

Two major steps in the modelling must be analyzed: the detection and the treatment of every contact.

The detection is straightforward in the case of circular particles. We define the distance $D_{j k}$ between two particles $j$ and $k$ by:

$$
D_{j k}(q)=\left|q_{k}-q_{j}\right|-\left(r_{j}+r_{k}\right)
$$

where ${ }^{\mathrm{t}} q=\left({ }^{\mathrm{t}} q_{1},{ }^{\mathrm{t}} q_{2}, \ldots,{ }^{\mathrm{t}} q_{n}\right)$ and $\left|q_{k}-q_{j}\right|=$
$\sqrt{\left(q_{k}^{x}-q_{j}^{x}\right)^{2}+\left(q_{k}^{y}-q_{j}^{y}\right)^{2}}$.

There is a contact between particles $j$ and $k$ when $D_{j k}(q)=0$, and an overlap when $D_{j k}(q)<0$. Efficient techniques for detecting contacts $[30,35]$ or closest neighbours are necessary when the number of particles increases in order to reduce the computational time.

Concerning the local treatment of contacts, the model proposed by Frémond $[26,32]$ has been retained. This original model is based on the theory of rigid bodies collisions and takes place in the line of the works of Moreau [33]. The numerical aspects were later developed in [36, 37].

Let us consider the set of $n$ particles as a deformable system composed of $n$ rigid solids. The motion equations of the system are:

$$
\left\{\begin{array}{l}
\mathbf{M} \boldsymbol{u}(t)=\boldsymbol{f}^{\mathrm{int}}(t)+\boldsymbol{f}^{\mathrm{ext}}(t) \quad \text { almost everywhere } \\
\mathbf{M}\left(\boldsymbol{u}^{+}(t)-\boldsymbol{u}^{-}(t)\right)=\boldsymbol{p}^{\mathrm{int}}(t)+\boldsymbol{p}^{\mathrm{ext}}(t) \text { everywhere }
\end{array}\right.
$$

where $\mathbf{M}$ is the $2 n \times 2 n$ mass matrix of the particles; $\boldsymbol{u}$ is the velocity vector of dimension $2 n,{ }^{\mathrm{t}} \boldsymbol{u}=$ $\left({ }^{\mathrm{t}} \boldsymbol{u}_{1},{ }^{\mathrm{t}} \boldsymbol{u}_{2}, \ldots,{ }^{\mathrm{t}} \boldsymbol{u}_{n}\right)$; the indices - and + refer to values before and after collisions; $f^{\text {ext }}$ (resp. $f^{\text {int }}$ ) is the vector of dimension $2 n$ of exterior forces (resp. interior forces) applied to the system.

Equation (2) applies almost everywhere except at the instant of the collision and is replaced by Equation (3). When contact is detected, velocities of colliding particles are discontinuous and we introduce in Equation (3) interior $\boldsymbol{p}^{\text {int }}$ and exterior $\boldsymbol{p}^{\text {ext }}$ percussions of the system. By definition, percussions have the dimension of a force multiplied by a time. The $\boldsymbol{p}^{\text {int }}$ percussions are unknown; they take into account the dissipative interactions between the particles which are colliding (dissipative percussions), and the reaction forces in order to avoid overlapping among particles (reactive percussions). Frémond [26,32] showed that these interior percussions are defined in duality with the velocity of deformation at the moment of impact $\frac{\boldsymbol{\Delta}\left(\boldsymbol{u}^{+}\right)+\boldsymbol{\Delta}\left(\boldsymbol{u}^{-}\right)}{2}$, in the sense of the work of internal forces. $\boldsymbol{p}^{\text {int }}$ depends on $\frac{\boldsymbol{\Delta}\left(\boldsymbol{u}^{+}\right)+\boldsymbol{\Delta}\left(\boldsymbol{u}^{-}\right)}{2}$ and Frémond used a pseudopotential of dissipation $\Phi$ (convex function [38]) defined as: $\Phi=\Phi^{\text {diss }}+\Phi^{\text {reac }}$ where $\Phi^{\text {diss }}$ and $\Phi^{\text {reac }}$ are two pseudopotentials which allow to define the dissipative and reactive interior percussions respectively, for their expression:

$$
\boldsymbol{p}^{\mathrm{int}} \in \partial \Phi\left(\frac{\boldsymbol{\Delta}\left(\boldsymbol{u}^{+}\right)+\boldsymbol{\Delta}\left(\boldsymbol{u}^{-}\right)}{2}\right)
$$

where the operator $\partial$ is the subdifferential which generalizes the derivative for convex functions [32]. The pseudopotential $\Phi^{\text {diss }}$ is chosen to be quadratic: $\Phi^{\text {diss }}=$ $\frac{k}{2}\left(\frac{\boldsymbol{\Delta}\left(\boldsymbol{u}^{+}\right)+\boldsymbol{\Delta}\left(\boldsymbol{u}^{-}\right)}{2}\right)^{2}$.

To find the velocity after collision of grains $\boldsymbol{u}^{+}$, we have to solve the following constrained minimization problem:

$$
\begin{aligned}
& \boldsymbol{v}=\underset{\boldsymbol{w} \in \boldsymbol{R}^{2 n}}{\arg \min }\left[{ }^{t} \boldsymbol{w} \mathbf{M} \boldsymbol{w}+\right. \Phi(\boldsymbol{\Delta}(\boldsymbol{w})) \\
&\left.-{ }^{t}\left(2 \boldsymbol{u}^{-}+\mathbf{M}^{-\mathbf{1}} \boldsymbol{p}^{\mathrm{ext}}\right) \mathbf{M} \boldsymbol{w}\right]
\end{aligned}
$$

where the solution $\boldsymbol{v}=\frac{\boldsymbol{u}^{+}+\boldsymbol{u}^{-}}{2}$.

The existence and uniqueness of the solution are demonstrated in $[26,32,37]$ and the minimization problem (5) is numerically solved using a time-stepping scheme and the classical Uzawa algorithm [37].

\subsection{Adaptation of the granular model to the crowd}

A pedestrian can be represented as a grain by giving it a willingness, i.e. a desire to move in a particular direction with a specific speed at each instant. In order to define the desired trajectory of one pedestrian, several strategies can be chosen: either the most comfortable trajectory for him or the shortest path or the fastest path to move from one place to another [14].

The desired direction $\boldsymbol{e}_{d, j}$ of an individual $j$ depends on the evolution space (obstacles, etc.), the time and also the characteristics of the individual (gender, age, hurried steps or not, etc.). It is defined by: $\boldsymbol{e}_{d, j}=\frac{\boldsymbol{u}_{d, j(t)}}{\left\|\boldsymbol{u}_{d, j}\right\|}$, where $\boldsymbol{u}_{d, j}$ is the desired velocity of the $j$ th pedestrian. The strategy of the shortest path to get from one point to another [39] is implemented through a Fast Marching algorithm and allows us to find $\boldsymbol{e}_{d, j}$.

The amplitude $\left\|\boldsymbol{u}_{d, j}\right\|$ of the desired velocity represents the speed at which the $j$ th pedestrian wants to move on the considered structure. It depends on the desired step length $L_{\mathrm{step}, 0, j}$ of the $j$ th pedestrian and on the lateral angular frequency $\omega_{j}$ of his free desired walking [1]:

$$
\left\|\boldsymbol{u}_{d, j}\right\|=\frac{L_{\text {step }, 0, j}}{\pi} \omega_{j}
$$




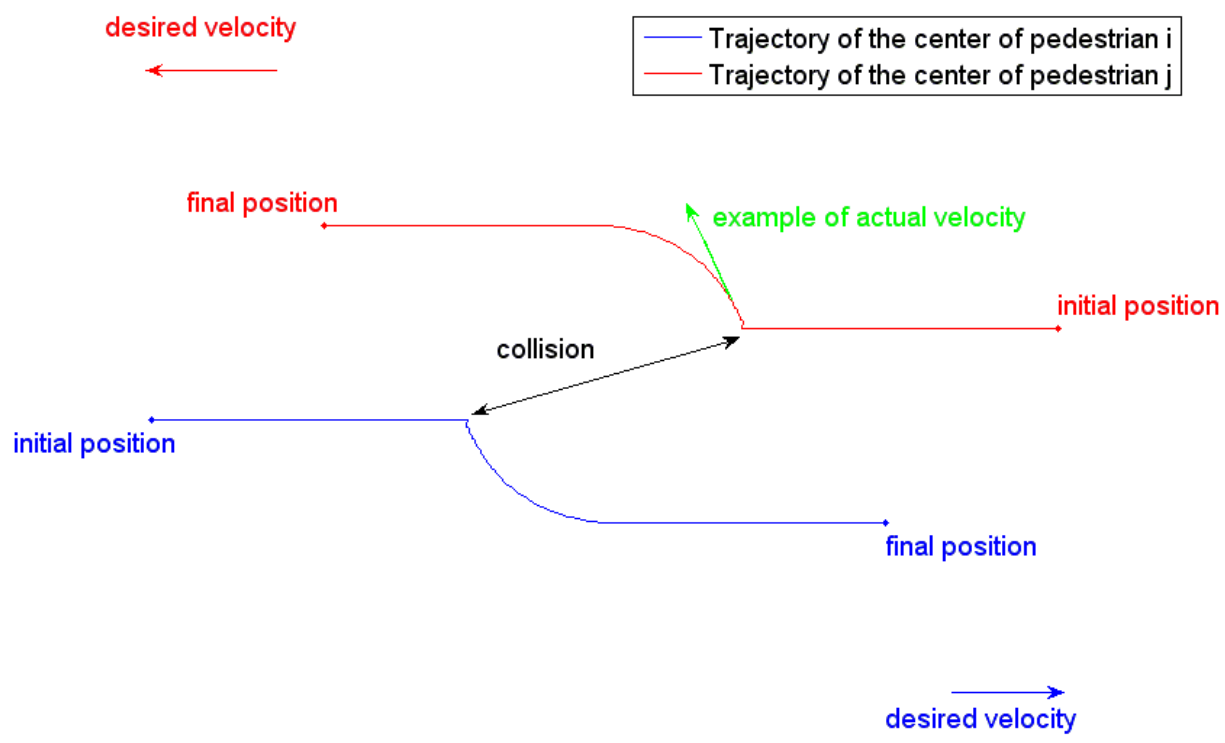

Fig. 1. Representation of the trajectories of two identical pedestrians $i$ and $j$ moving into opposite directions, $\tau_{i}=\tau_{j}=2 \mathrm{~s}$. After the collision, for each pedestrian, the external acceleration force allows pedestrian to gradually switch from the actual velocity after choc to the desired velocity.

To adapt Frémond's model to the crowd, we introduce an external acceleration force $\boldsymbol{f}_{\mathrm{a}}^{\mathrm{ext}}(t)[15]$ that gives a desired direction and intensity of the velocity to each pedestrian (Fig. 1). The generic component $\boldsymbol{f}_{\mathrm{a}, j}^{\text {ext }}(t)$ of the force: ${ }^{\mathrm{t}} \boldsymbol{f}_{\mathrm{a}}^{\text {ext }}=\left({ }^{\mathrm{t}} \boldsymbol{f}_{\mathrm{a}, 1}^{\text {ext }}, \boldsymbol{f}_{\mathrm{a}, 2}^{\text {ext }}, \ldots,{ }^{\mathrm{t}} \boldsymbol{f}_{\mathrm{a}, n}^{\mathrm{ext}}\right)$ of dimension $2 n$, is associated with pedestrian $j$ and can be expressed as:

$$
\boldsymbol{f}_{\mathrm{a}, j}^{\mathrm{ext}}(t)=m_{j} \frac{\left\|\boldsymbol{u}_{d, j}\right\| \boldsymbol{e}_{d, j}(t)-\boldsymbol{u}_{j}(t)}{\tau_{j}}
$$

where $\boldsymbol{u}_{j}$ is the actual velocity; $\tau_{j}$ is a relaxation time, allowing to recover the desired velocity after a contact. Smaller values of $\tau_{j}$ let the pedestrians walk more aggressively [40]. For numerical simulations, $\tau_{j}$ is taken equal to $2 \mathrm{~s}$.

The pedestrians' behavior can be enriched with the addition of other external social forces [41] in order to become more realistic.

\subsection{Simulations}

Some self-organisation phenomena occurring in pedestrian crowds, already analyzed in $[9,11]$, can also be reproduced by the adapted Frémond's model. This paper will focus on two phenomena: the occurrence of lanes of uniform walking direction in crowd of pedestrians moving into opposite directions and the formation of pedestrian arches around an exit in the case of a panic escape of confined pedestrians. In both cases, we choose that the desired velocity of each $j$ th pedestrian follows a Gaussian distribution of average $1.34 \mathrm{~m} . \mathrm{s}^{-1}$ and of standard deviation $0.26 \mathrm{~m} . \mathrm{s}^{-1}[10]$. This corresponds to a normal walking velocity.

For the first phenomenon, we simulate a melee of 1500 pedestrians. Initially, the pedestrians are randomly

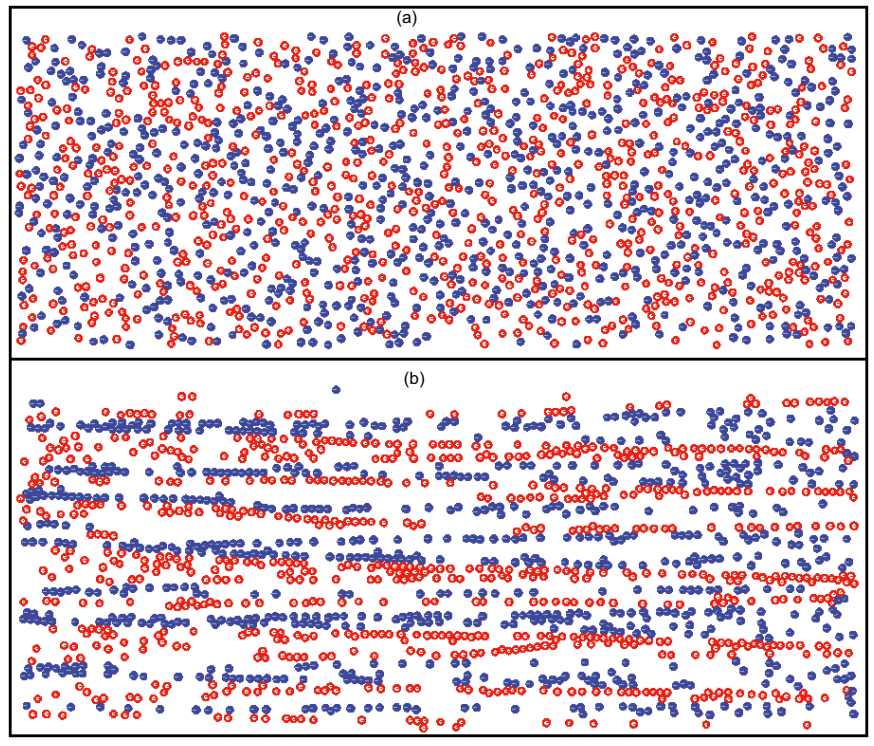

Fig. 2. Adapted Frémond's model - simulation of a melee with 1500 pedestrians - (a) at time $t=0 \mathrm{~s}$, (b) at time $t=40 \mathrm{~s}$.

positioned in a rectangular domain of $80 \mathrm{~m}$ in length and $25 \mathrm{~m}$ in width. 750 pedestrians in filled blue circles want to move to the left and 750 others in empty red circles to the right (Fig. 2). To keep the number of pedestrians walking on the considered domain constant, once a pedestrian exits the room on one side, a similar pedestrian enters the room with a random position along the $y$-axis on the other side.

We can observe the formation of counter flow lines where pedestrians do not keep some distance between them. The number of forming lanes depends on the width of the walkway and on the pedestrian density $[9,15,41]$. 


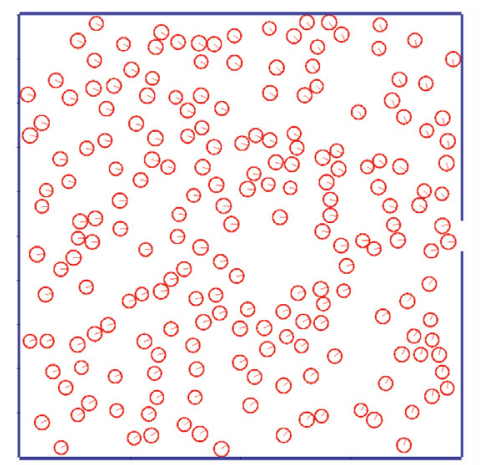

(a)

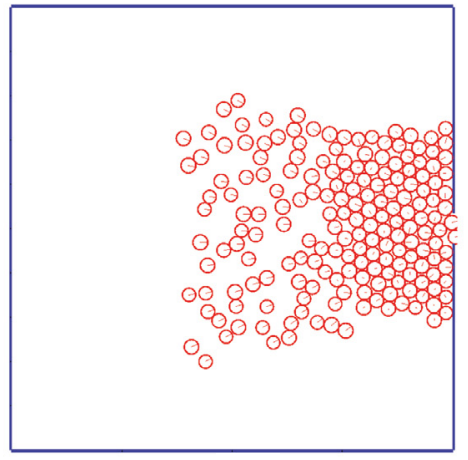

(b)

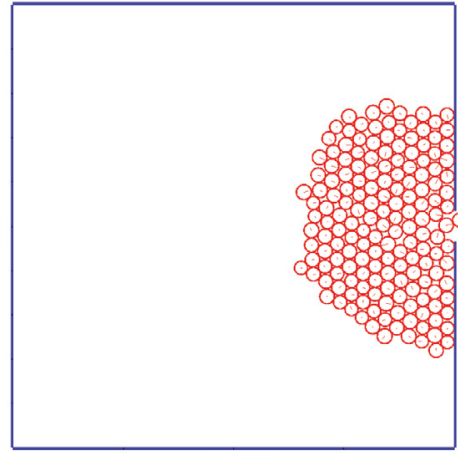

(c)

Fig. 3. Adapted Frémond's model - simulation of a room evacuation - formation of an arch $-(\mathrm{a})$ at time $t=0 \mathrm{~s}$ with 200 pedestrians, (b) at time $t=6 \mathrm{~s}$ with 187 pedestrians, (c) at time $t=12 \mathrm{~s}$ with 166 pedestrians.

For the second phenomenon, we consider a square room of side $20 \mathrm{~m}$, where 200 pedestrians want to escape by the right side (Fig. 3 ).

We observe the formation of an arch as it has been experimentally observed in the case of mice in an emergency situation. In our case of an evacuation with normal walking velocity, regular exterior forces have to be added to the adapted Frémond's model, as in Helbing's model $[9,15,40]$, in order to obtain a more realistic simulation with pedestrians keeping some distance between them when their density is not too high.

\section{Crowd-structure interaction}

In this section, the model previously presented for the crowd is enhanced in order to manage the crowd-structure interaction. The final model must be able to represent the behavior of the footbridge, each pedestrian $j$ and the pedestrian-structure coupling.

It is first assumed that the floor is flat and horizontal; the main axis (longitudinal) of the bridge is straight, so we define a Cartesian coordinate system with $x$-axis which is parallel to the main axis, the $z$-axis vertical and the $y$-axis oriented accordingly; the floor oscillation takes place in the horizontal plane ( $x y$-plane); its longitudinal oscillation is negligible and its displacements are supposed constant along the $y$-axis; the pedestrians are walking in the $x y$-plane. Only the pedestrian and bridge lateral oscillations are considered and the $x, y$ and $z$ displacement components are then assumed to be decoupled. We assume finally that the forces applied by the two legs of one pedestrian to the floor are identical.

The first section is devoted to the presentation of the differential equations governing the crowd-structure interaction. A model similar to the one proposed by Bodgi [6] is used, consisting of $n+1$ equations: one of the dynamic of the structure with excitation due to pedestrian and, for each pedestrian one differential equation of Kuramoto [2] relative to his walking oscillations. In the second section, the whole model is applied to the walking of pedestrians on the north span of the Millennium Footbridge in London.

\subsection{Coupling model}

Let $U_{y}\left(q^{x}, t\right)$ be the footbridge lateral displacement at position $q^{x}$ along $x$-axis and at time $t$. The projection onto the first lateral mode shape of the footbridge leads us to write: $U_{y}\left(q^{x}, t\right)=U(t) \psi_{1}\left(q^{x}\right)$ where $U(t)=A(t) \sin \left(\psi_{\text {str }}(t)\right)$ is the lateral modal displacement of the footbridge with total phase $\psi_{\text {str }}(t)$ and amplitude $A(t)$, and $\psi_{1}\left(q^{x}\right)$ is the first lateral modal shape of the footbridge, with $\max \left(\psi_{1}\left(q^{x}\right)\right)=1$. The displacement history $U(t)$ is assumed to be a chirp: $A(t)$ is a slowly varying quantity when compared to $\psi_{\text {str }}(t)$, then the time derivative of $U$ can be approximated by: $\dot{U}(t) \simeq$ $A(t) \omega_{\text {str }} \cos \left(\psi_{\text {str }}(t)\right)$ where $\omega_{\text {str }}$ is the angular frequency of the first lateral mode of the structure, and $A(t)$ can be expressed by $A(t)=\sqrt{U^{2}(t)+\frac{\dot{U}^{2}(t)}{\omega_{\text {str }}^{2}}}$.

To take into account the oscillations of the structure in the crowd movement model, the amplitude of the desired velocity of the $j$ th pedestrian in equation (6) is modified and will be noted $\left\|\boldsymbol{u}_{d, j}\right\|_{\bmod }(t)$. It depends on $A(t)$ and $\psi_{\text {str }}(t)$ through the instantaneous step length $L_{\text {step }, j}(t)$ and the instantaneous angular frequency $\dot{\phi}_{j}(t)$ of the $j$ th pedestrian:

$$
\left\|\boldsymbol{u}_{d, j}\right\|_{\bmod }(t)=\frac{L_{\mathrm{step}, j}(t)}{\pi} \dot{\phi}_{j}(t)
$$

and $L_{\mathrm{step}, j}(t)$ is chosen dependent on $A(t)$ :

$$
L_{\text {step }, j}(t)=L_{\text {step }, 0, j} \max \left(1-\frac{A(t)}{A_{\max , \mathrm{j}}}, 0\right)
$$

where $A_{\max , j}$ is the maximum amplitude of the lateral displacement of the footbridge that the $j$ th pedestrian can tolerate. When $A$ reaches $A_{\max , j}$, oscillations are so important that the $j$ th pedestrian stops walking.

We choose a differential equation of Kuramoto [1,2] to govern the evolution of the total phase $\phi_{j}(t)$ of the walking force generated by the $j$ th pedestrian on the bridge:

$$
\dot{\phi}_{j}(t)=\omega_{j}+C A(t) \sin \left(\psi_{\mathrm{str}}(t)-\phi_{j}(t)+\frac{\pi}{2}\right)
$$


where $C$ quantifies pedestrians' sensitivity to bridge vibrations.

In Equation (10), pedestrians are sensitive to footbridge displacement amplitude $A(t)$ as proposed in [2]; it should be noted that in [1], they are sensitive to footbridge acceleration.

Synchronization can be seen as an adaptation of the frequency of the force generated by a pedestrian to the frequency of the structure; hence, the use of a differential equation which allows the instantaneous angular frequency of the pedestrian to converge to that of the structure. For a walking on a rigid floor, this equation is reduced to: $\dot{\phi}_{j}(t)=\omega_{j}$.

Finally, the equation which describes the dynamic of the footbridge projected onto its first mode shape is:

$$
\begin{array}{r}
{\left[M_{\mathrm{str}}+\sum_{j=1}^{n} m_{j} \psi_{1}^{2}\left(q_{j}^{x}(t)\right)\right] \ddot{U}(t)+C_{\mathrm{str}} \dot{U}(t)+K_{\mathrm{str}} U(t)=} \\
\sum_{j=1}^{n} \psi_{1}\left(q_{j}^{x}(t)\right) F_{j}(t)
\end{array}
$$

with the following initial conditions, at time $t=t_{0}$ : $U_{0}=U\left(t_{0}\right), \dot{U}_{0}=\dot{U}_{0}\left(t_{0}\right)$ and where $M_{\text {str }}, C_{\text {str }}$ and $K_{\text {str }}$ are the modal (generalized) mass, damping and stiffness of the lateral first mode of the footbridge, respectively; $m_{j}$ the mass of the $j$ th pedestrian; $n$ the number of pedestrians on the footbridge; $F_{j}$ the amplitude of the total lateral force applied by the $j$ th pedestrian to the footbridge.

As each pedestrian is walking in the $x y$-plane, the force applied by the $j$ th pedestrian on the floor includes a normal $F_{N, j}$ and a tangential $F_{T, j}$ component to the pedestrian's motion. We assume that each pedestrian imparts an alternating (sinusoidal) sideways force to the bridge [2]. As the frequency of $F_{T, j}$ is usually considered as twice the frequency of $F_{N, j}$ [1], the expression of $F_{j}$ can be written as follows:

$$
\begin{aligned}
F_{j}(t) & ={ }^{\mathrm{t}}\left(F_{T, j}(t) \boldsymbol{e}_{j}(t)+F_{N, j}(t) \boldsymbol{e}_{j}^{\perp}(t)\right) \boldsymbol{e}_{y} \\
& ={ }^{\mathrm{t}}\left(T_{j} \sin \left(2 \phi_{j}(t)\right) \mathbf{e}_{j}(t)+N_{j} \sin \left(\phi_{j}(t)\right) \boldsymbol{e}_{j}^{\perp}(t)\right) \boldsymbol{e}_{y} \\
& =T_{j} \sin \left(2 \phi_{j}(t)\right) \sin \left(\theta_{j}(t)\right)+N_{j} \sin \left(\phi_{j}(t)\right) \cos \left(\theta_{j}(t)\right)
\end{aligned}
$$

where $T_{j}$ (resp. $N_{j}$ ) is the maximum amplitude of $F_{\mathrm{T}, j}$ (resp. $F_{\mathrm{N}, j}$ ); $\boldsymbol{e}_{j}$ (resp. $\boldsymbol{e}_{j}^{\perp}$ ) is the unit direction vector of the $j$ th pedestrian's motion (resp. normal to the $j$ th pedestrian's motion), $\boldsymbol{e}_{j}=\frac{\boldsymbol{u}_{j}(t)}{\left\|\boldsymbol{u}_{j}\right\|} ; \boldsymbol{e}_{y}$ is the unit direction vector of the lateral motion of the footbridge; $\theta_{j}(t)$ is the angle between the direction of the $j$ th pedestrian's motion and the direction of the longitudinal axis of the bridge.
To solve the previous system of differential equations, equations (10) and (11), a state representation is first used by introducing the state vector $\boldsymbol{z}={ }^{t}\left(U, V, \phi_{1}, \ldots, \phi_{n}\right)$. We get:

$$
\dot{\boldsymbol{z}}=\left[\begin{array}{c}
\dot{U} \\
\dot{V} \\
\dot{\phi_{1}} \\
\cdots \\
\dot{\phi_{n}}
\end{array}\right]=\left[\begin{array}{c}
V \\
\frac{\sum_{j=1}^{n} \psi_{1}\left(q_{j}^{x}(t)\right) F_{j}(t)-C_{\mathrm{str}} \dot{U}(t)-K_{\mathrm{str}} U(t)}{M_{\mathrm{str}}+\sum_{j=1}^{n} m_{j} \psi_{1}^{2}\left(q_{j}^{x}(t)\right)} \\
\omega_{1}+C A(t) \sin \left(\psi_{\mathrm{str}}(t)-\phi_{1}(t)+\frac{\pi}{2}\right) \\
\cdots \\
\omega_{n}+C A(t) \sin \left(\psi_{\mathrm{str}}(t)-\phi_{n}(t)+\frac{\pi}{2}\right)
\end{array}\right]
$$

Then, the explicit method of Runge-Kutta of 2nd and 3rd order based on the Bogacki-Shampine method is applied to Equation (13) (ode23 toolbox of the MATLAB software).

\subsection{Numerical simulations}

In this section, we apply the presented model to simulate the pedestrians' walking on the north span of the Millennium Footbridge in London, of length $L=81 \mathrm{~m}$ and width is $l=4 \mathrm{~m}$. The values of the model parameters are: $r_{j}$ is chosen randomly between $[0.3 \mathrm{~m}, 0.35 \mathrm{~m}]$ which represents the case of a pedestrian warmly dressed with a backpack [18], $m_{j}=230 \pi r_{j}^{2}$ (in kg), $L_{\text {step }, 0, j}$ follows a normal distribution with mean value $0.71 \mathrm{~m}$ and standard deviation $0.071 \mathrm{~m} \mathrm{[42],} N_{j}=35 \mathrm{~N}[1,43]$, $T_{j}=120 \mathrm{~N}[43], M_{\text {str }}=113000 \mathrm{~kg}, C_{\text {str }}=11000 \mathrm{~kg} . \mathrm{s}^{-1}$, $K_{\text {str }}=4730000 \mathrm{~kg} . \mathrm{s}^{-2}, \omega_{j}$ follows a normal distribu-

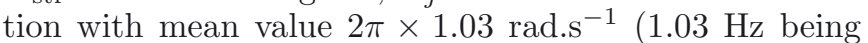
the modal frequency of the north span) and standard deviation $2 \pi \times 0.094 \mathrm{rad} . \mathrm{s}^{-1}, \phi_{j}$ is chosen randomly between $[-\pi, \pi]$ with mean value zero, $U_{0}=0, \dot{U}_{0}=0$, $A_{\max , j}=0.2 \mathrm{~m}, C \simeq 16 \mathrm{~m}^{-1} \cdot \mathrm{s}^{-1}$ [2]. As in [2], the first lateral modal shape is neglected: $\psi_{1}\left(q_{j}^{x}(t)\right)=1$.

When few pedestrians are walking on the footbridge (about 100, Fig. 4a), the lateral displacement of the structure stays small (Fig. 4d). Only some pedestrians are synchronized with the footbridge displacement (Figs. 4b and $4 \mathrm{c}$ ), but not enough to trigger the phenomenon of synchronization.

To estimate the degree of coherence of the pedestrian phase, we compute then the order parameter $\Gamma(t)$ introduced in [2]:

$$
\Gamma(t)=\frac{1}{n}\left|\sum_{j=1}^{n} \mathrm{e}^{i \phi_{j}(t)}\right|
$$

An increase of this parameter reflects the fact that pedestrians synchronize one by one with the structure to reach a maximum number of synchronized pedestrians $(\Gamma=1)$. For each number of pedestrians on the bridge, when there is synchronization, the calculation of the order parameter gives the lower limit of percentage of synchronized 
(a)

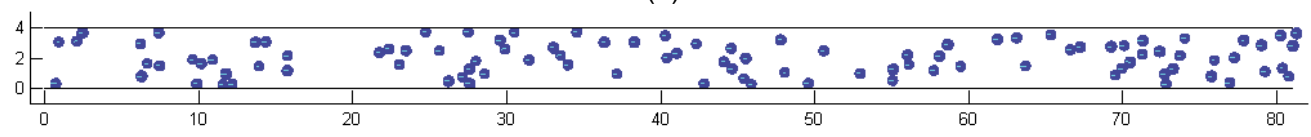

(b)

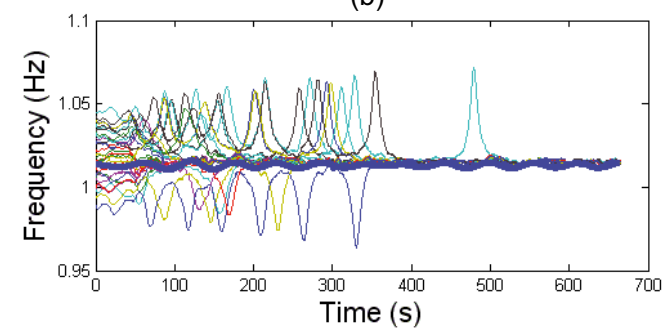

(c)

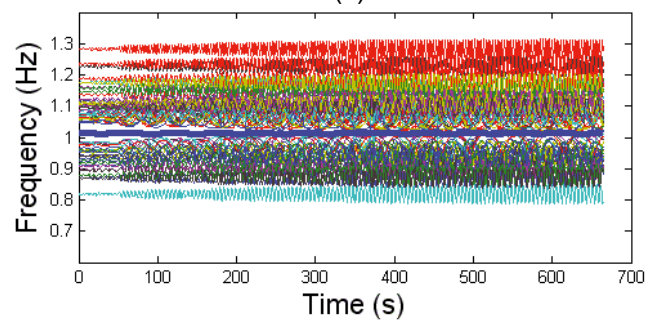

(d)

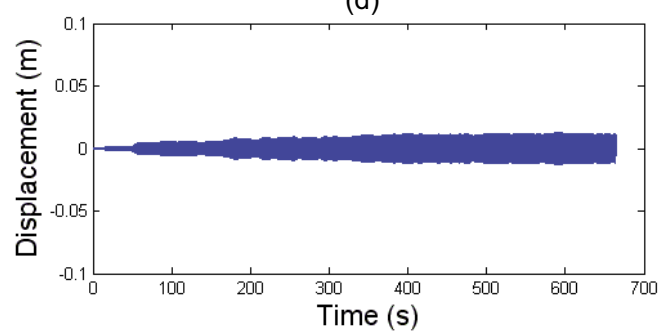

(e)

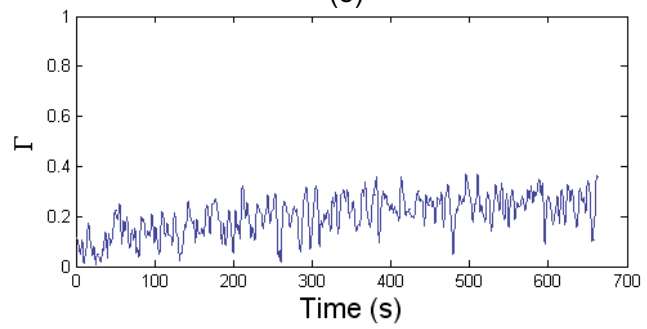

Fig. 4. A crowd of 100 pedestrians crossing the north span of Millenium bridge - full model (adapted Frémond's model + Kuramoto equations) - (a) representation of the 100 pedestrians on the footbridge; (b) time evolution of the instantaneous frequency $\frac{\dot{\phi}_{j}(t)}{2 \pi}$ for synchronized pedestrians (30) with the structure. The blue curve in bold represents the instantaneous frequency of the loaded structure; (c) time evolution of the instantaneous frequency $\frac{\dot{\phi}_{j}(t)}{2 \pi}$ for not synchronized pedestrians $(70)$ with the structure; (d) time evolution of the lateral displacement of the structure $U(t) ;(\mathrm{e})$ time evolution of the order parameter.

pedestrians. Figure 4e shows that the order parameter for the 100 pedestrians remains low and therefore the phenomenon of synchronization is not triggered.

Conversely, when the number of pedestrians is high enough, for instance about 180 pedestrians (Fig. 5a), the lateral displacement of the footbridge increases when the phenomenon of synchronization is triggered (Figs. $5 \mathrm{~d}$ and e). 170 pedestrians are synchronized with the footbridge displacement (Figs. 5b and c). In Figure 5d, the amplitude of the lateral footbridge's displacement reaches a threshold after a transition period. In Figure 5e, we note that the evolution of the order parameter is consistent with that of the amplitude of lateral displacement of the bridge (Fig. 5d).

After several simulations of our 2D model, the critical number of pedestrians, i.e. the minimal number of pedestrians walking on the bridge triggering the phenomenon of synchronisation, is numerically estimated between 160 and 180. These values are in agreement with the results found experimentally by Arup [44,45] and numerically by $[1,2]$. In $[1,2]$ the authors proposed also an analytical expression of the critical number, depending on the maximum lateral force transmitted by the pedestrians, on a pedestrians' sensitivity parameter to the bridge vibrations and on the bridge mechanical and geometrical characteristics.

\section{Conclusion}

This paper presented a 2D coupled model for describing the interactions between pedestrians and a structure. In the first part of the paper, the $2 \mathrm{D}$ displacement of each pedestrian on a rigid floor is handled by an adapted granular media model based on the theory of collisions of rigid bodies developed by Frémond. In the second part, the pedestrian-structure interaction is described with particular attention to the walking lateral oscillation of each pedestrian. Simulations with the full model allowed us to reproduce some existing numerical and experimental results: the formation of counter flow lines and the phenomenon of arches in the case of rigid floors, without crowd-structure interaction, and the phenomenon of locking when crowd-structure interaction is taken into account.

These encouraging results should be further validated by applying the proposed model to other structures than the Millenium bridge. Moreover, an interesting characteristic of the proposed model is the possibility of studing the phenomenon of pedestrian-pedestrian synchronization depending on the density of pedestrians, and evaluating its influence on the phenomenon of crowd-structure synchronization. 
(a)

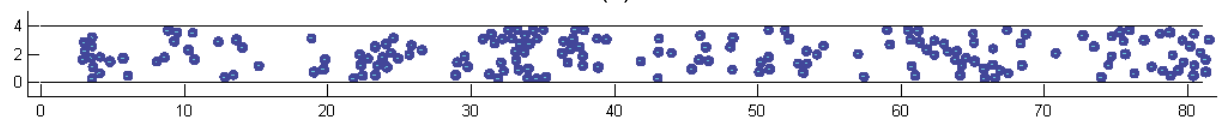

(b)

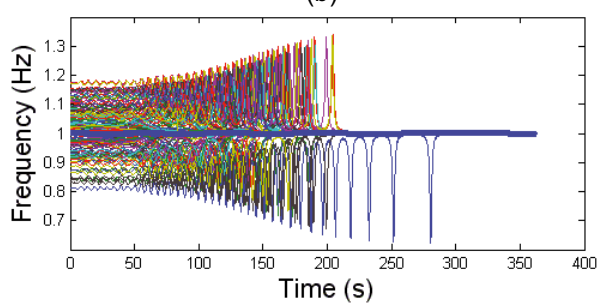

(c)

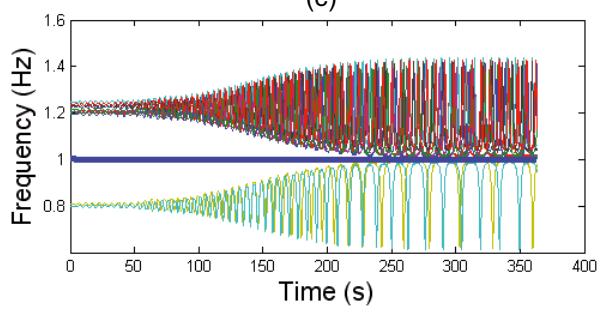

(d)

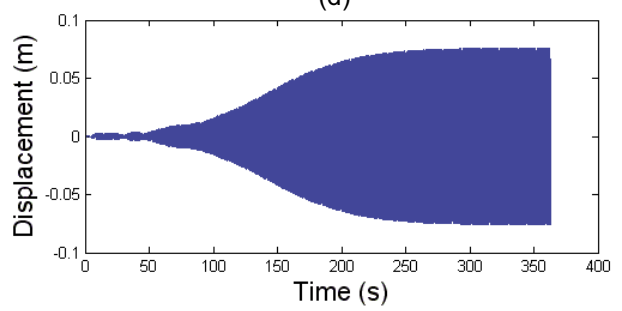

(e)

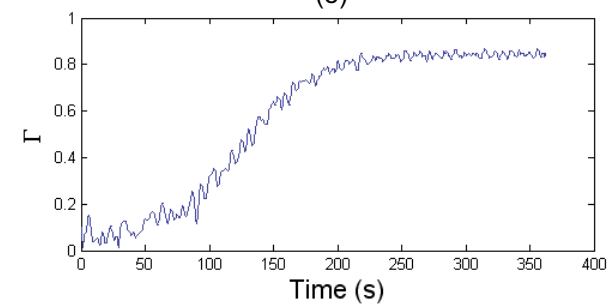

Fig. 5. A crowd of 180 pedestrians crossing the north span of Millenium bridge - full model (adapted Frémond's model + Kuramoto equations) - (a) representation of the 180 pedestrians on the footbridge; (b) time evolution of the instantaneous frequency $\frac{\dot{\phi}_{j}(t)}{2 \pi}$ for synchronized pedestrians (170) with the structure. The blue curve in bold represents the instantaneous frequency of the loaded structure; (c) time evolution of the instantaneous frequency $\frac{\dot{\phi}_{j}(t)}{2 \pi}$ for not synchronized pedestrians (10) with the structure; (d) time evolution of the lateral displacement of the structure $U(t)$; (e) time evolution of the order parameter.

Acknowledgements. The authors would like to thank Michel Frémond, Professor at Roma II University, for his fruitful suggestions and pertinent remarks about this manuscript.

\section{References}

[1] J. Bodgi, Synchronisation piétons-structure: Application aux vibrations des passerelles souples, Ph.D. thesis, École Nationale des Ponts et Chaussées, 2008

[2] S. Strogatz, D. Abrams, A. McRobie, B. Eckhardt, E. Ott, Theoretical mechanics: Crowd synchrony on the Millenium bridge, Nature 438 (2005) 43-44

[3] S. Zivanovic, A. Pavic, P. Reynolds, Vibration serviceability of footbridges under human-induced excitation: a litterature review, J. Sound Vib. 279 (2005) 1-74

[4] S. Zivanovic, V. Ravic, I. El-Bahnasy, A. Pavic, Statistical characterisation of parameters defining human walking as observed on an indoor passerelle, in Experimental Vibration Analysis for Civil Engineering Structures, EVACES'07, Porto, Portugal, 219-225 (2007)

[5] D.M. Abrams, Two coupled oscillator models: the Millenium bridge and the chimera state, Ph.D. thesis, Cornell University, 2006

[6] J. Bodgi, S. Erlicher, P. Argoul, Lateral vibration of footbridges under crowd-loading: continuous crowd modelling approach, Key Engineering Materials 347 (2007) 685-690
[7] P. Charles, C. Delavaud, A. Hekimian, J. Renault, T. Saez, Dispositif d'essais sur un modèle de passerelle, rapport d'essais SETRA, 2005

[8] B.D. Hankin, R.A. Wright, Passenger flow in subways, Oper. Res. 9 (1958) 81-88

[9] D. Helbing, Traffic and related self-driven many-particle systems, Rev. Mod. Phys. 73 (2002) 1067-1141

[10] L.F. Henderson, The statistics of crowd fluids, Nature 229 (1971) 381-383

[11] J. Venel, Modélisation mathématique des mouvements de foule, Ph.D. thesis, Laboratoire de Mathématiques, Université Paris XI, Orsay, France, 2008

[12] V. Blue, J. Adler, Cellular automata microsimulation of bi-directional pedestrian flows, J. Transp. Res. Board 1678 (2000) 135-141

[13] K. Teknomo, Application of microscopic pedestrian simulation model, Transp. Res. Part F 9 (2006) 15-27

[14] S.P. Hoogendoorn, P.H.L. Bovy, W. Daamen, Microscopic pedestrian wayfinding and dynamics modelling, Pedestrian and Evacuation Dynamics (2001) 123-154

[15] D. Helbing, P. Molnar, Social force model for pedestrian dynamics, Phys. Rev. E 51 (1995) 4282-4286

[16] C. Reynolds, Flocks, herds, and schools: A distributed behavioral model, Comput. Graph. 21 (1987) 25-34

[17] S. Paris, J. Pettré, S. Donikian, Pedestrian reactive navigation for crowd simulation: a predictive approach, Comput. Graph. Forum 26 (2007) 665-674 
[18] S. Paris, Caractérisation des niveaux de services et modélisation des circulations de personnes dans les lieux d'échanges, Ph.D. thesis, Université de Rennes 1, 2007

[19] M.S. Garcia, Stability, scaling and chaos in passivedynamic gait models, Ph.D. thesis, Cornell University, 1999

[20] S. Erlicher, A. Trovato, P. Argoul, Modeling the lateral pedestrian force on a rigid floor by a self-sustained oscillator, Mech. Syst. Signal Process 2010 doi:10.1016/j.ymssp.2009.11.006

[21] P.A. Cundall, A computer model for simulating progressive large scale movements of blocky rock systems, in Proc. Symp. Int. Soc. Rock Mech., 1971, Vol. 1

[22] P.A. Cundall, O.D.L. Strack, A discrete numerical model for granular assemblies, Geotechnique 29 (1979) 47-65

[23] Y. Kishino, Disk model analysis of granular media, Micromechanics of Granular Materials (1988) 143-152

[24] M.P. Allen, D.J. Tildesley, Computer simulation of liquids, Oxford University Press, 1987

[25] M. Jean, J.J. Moreau, Unilaterality and dry friction in the dynamics of rigid bodies collection, Contact Mechanics Int. Symp. (1992) 31-48

[26] M. Frémond, Rigid bodies collisions, Phys. Lett. A 204 (1995) 33-41

[27] F. Radjai, M. Jean, J.J. Moreau, S. Roux, Force distributions in dense two-dimensional granular systems, Phys. Rev. Lett. 77 (1996) 264-277

[28] M. Jean, The non smooth contact dynamics method, Compt. Methods Appl. Math. Eng. 177 (1999) 235-257

[29] L. Paoli, Time discretization of vibro-impact, Phil. Trans. R. Soc. A 359 (2001) 2405-2428

[30] M. Renouf, Optimisation numérique et calcul parallèle pour l'étude des milieux divisés bi- et tridimensionnels, Ph.D. thesis, Université Montpellier II, Sciences et Techniques du Languedoc, 2004

[31] G. Saussine, C. Cholet, P.E. Gautier, F. Dubois, C. Bohatier, J.J. Moreau, Modelling ballast behaviour under dynamic loading, Part 1: a 2d polygonal discrete element method approach, Comput. Meth. Appl. Mech. Eng. 195 (2006) 2841-2859

[32] M. Frémond, Collisions, Edizioni del Dipartimento di Ingegneria Civile dell'Università di Roma Tor Vergata, ISBN 978-88-6296-000-7, 2007
[33] J.J. Moreau, Unilateral contact and dry friction in finite freedom dynamics, in J.J. Moreau, P.-D. Panagiotopoulos (ed.) Non Smooth Mechanics and Applications, CISM Courses and Lectures, Vol. 302, Springer-Verlag, Wien, New York, 1988, pp. 1-82

[34] J.J. Moreau, Some numerical methods in multibody dynamics: Application to granular materials, Eur. J. Mech. A/Solids (1994) 93-114

[35] C. Ericson, Real Time Collision Detection, Morgan Haufmann Publishers, 2004

[36] S. Dal Pont, E. Dimnet, A theory for multiple collisions of rigid solids and numerical simulation of granular flow, Int. J. Solids Struct. 43/20 (2006) 6100-6114

[37] S. Dal Pont, E. Dimnet, Theoretical approach to instantaneous collisions and numerical simulation of granular media using the A-CD ${ }^{2}$ method, Communications in Applied Mathematics and Computational Science, Berkeley 3/1 (2008) 1-24

[38] J.J. Moreau, Sur les lois du frottement, de la viscosité et de la plasticité, C. R. Acad. Sci. Paris 271 (1970) 608-611

[39] R. Kimmel, J.A. Sethian, Fast marching methods for computing distance maps and shortest paths, Technical Report 669, CPAM, University of California, Berkeley, 1996

[40] D. Helbing, I. Farkas, T. Vicsek, Simulating dynamic features of escape panic, Nature 407 (2000) 487-490

[41] D. Helbing, I. Farkas, P. Molnár, T. Vicsek, Simulation of pedestrians crowds in normal and evacuation situations, M. Schreckenberg and S. Deo Sarma (Ed.), Pedestrian and evacuation dynamics, 2002, pp. 21-58

[42] S. Zivanovic, A. Pavic, P. Reynolds, Probability-based prediction of multi-mode vibration response to walking excitation, Eng. Struct. 29 (2007) 942-954

[43] T.P. Andriacchi, J.A. Ogle, J.O. Galante, Walking speed as a basis for normal and abnormal gait measurements, J. Biomech. 10 (1977) 261-268

[44] P. Dallard, A.J. Fitzpatrick, A. Flint, A. Low, R.M. Ridsdill-Smith, The Millenium bridge London - problems and solutions, The Structural Engineer 79 (2001a) 15-17

[45] P. Dallard, A.J. Fitzpatrick, A. Flint, A. Low, R.M. Ridsdill-Smith, The Millenium bridge London, The Structural Engineer 79 (2001b) 17-33 